Correo: innova@uide.edu.ec

\title{
Evaluación de las publicaciones realizadas en la revista INNOVA Research Journal del 2016
}

\section{Evaluation of the publications made in the 2016 INNOVA Research Journal}

Jorge Luis Vinueza-Martínez

Universidad Estatal de Milagro-UNEMI, Ecuador

Mirella Azucena Correa-Peralta

Universidad Estatal de Milagro-UNEMI, Ecuador

Autor para correspondencia: jvinuezam@unemi.edu.ec, mcorreap@unemi.edu.ec

Fecha de recepción: 26 de Junio de 2017 - Fecha de aceptación: 15 de Noviembre de 2017

\section{Resumen}

El desarrollo de este estudio es analizar la literatura de 12 publicaciones de la revista INNOVA Research Journal. La bibliografía fue tomada de forma directa del sitio web de la revista. La información de volumen, número, año, autores, palabras claves, título, referencia bibliográfica entre otras características se registró en una hoja electrónica para analizar e interpretar, se encontró 90 artículos publicados. Se evidenció que los autores que más publicaron fueron Guerrero Bejarano María, Nájera Santiago y Chavez Eras Andrés. El trabajo es auténtico y primero de este tipo para la revista.

Palabras clave: evaluación; publicaciones; revista INNOVA

\begin{abstract}
The development of this study is to analyze the literature of 12 publications of the INNOVA Research Journal. The bibliography was taken directly from the magazine's website. The information of volume, number, year, authors, keywords, title, bibliographic reference among other characteristics was recorded in an electronic sheet to analyze and interpret, found 90 published articles. It was evidence that the authors that most published were Guerrero Bejarano Maria, Nájera Santiago and Chavez Eras Andrés. The work is authentic and the first of its kind for the journal.
\end{abstract}

Key words: evaluation; publications; INNOVA journal. 


\section{Introducción}

La publicación científica es divulgar y escribir los resultados al final de un proceso de investigación científica; cada forma de publicación, tiene normas, políticas y definiciones diferentes que los autores deben conocer cómo escribir (Gallego Becerra, 2013), no es para el lauro personal sino para compartir el cúmulo de experiencias adquiridas (Zotta, 2015).

Cabe mencionar que la difusión científica es un pilar fundamental cognitivo para desarrollar futuras investigaciones y brindar la posibilidad de difundir trabajos a la comunidad y avance del conocimiento (Zotta, 2015)

Las publicaciones científicas o PC según Skeef citado por Mollo Pécora (1997) es el resultado de trabajos de investigación, sin embargo otros autores como Chauí (1994), Morales Morejón (1997) entre otros consideran a la PC como los aportes en términos de productividad científica que va más allá de la producción bibliográfica. Otros autores consideran como la esencia de las universidades que contribuye al desarrollo de la ciencia, creando en el investigador nuevos aspectos a descubrir sobre determinados aspectos y comprende: acumulación de información, desarrollo de teoría, validación y comprobación, documentar y difundir información a través de publicaciones, eventos científicos, tesis de grado, patentes, exposiciones científicas o documentos normativos (Piedra, Martínez, 2007).

En el Ecuador, el Consejo de evaluación, acreditación y aseguramiento de la calidad de la Educación Superior (CEAACES) está enfocado en elevar el nivel de investigación científica a nivel país y ha considerado indicadores para la producción científica, producción regional, libros o capítulos de libros. En este sentido la producción regional constituyen los artículos académicos y científicos en revistas que garanticen la calidad de las publicaciones, generalmente en revistas contenidas en base de datos de Latindex (catálogo), Scielo, Lylax, Redalyc, Ebsco, Proquest, Jstor, OAJI, Doaj y ponencias. (CEAACES, 2015), por esto las universidades se encuentran en este desafío de mejorar su producción investigativa de forma sustancial.

\section{Materiales y métodos}

Los resultados obtenidos fueron de manera directa en la revista Innova Research Journal http://www.journaluidegye.com/magazine/index.php/innova/, obteniéndose una población de 90 artículos. Para lo cual se realizó por fases:

- Identificar las publicaciones para la búsqueda de las publicaciones en la revista Innova

- Construir base de datos en hoja electrónica de las publicaciones en función de volumen, número, año, autores, palabras claves, título, número de páginas

- Analizar patrón de autoría y colaboradores de las publicaciones

- Validar y evaluar información agrupando las palabras claves en subtemas

- Consultar los artículos en google académico para obtener si han sido citados por artículos

\section{Resultados}

La revista Innova Research Journal catalogado de forma mensual en Latindex con ISSN 2477-9024 basado en los subtemas de ciencias de la comunicación, ciencias sociales y 
humanidades, educación, relaciones internacionales inició en el 2016 (Latindex, 2015), con 90 artículos publicados en ese año (Ver Tabla 1). Publicó 268 keywords en relación a educación, administración, psicología, tecnología, talento humano; la palabra clave de liderazgo se presenta en 13 artículos, seguido por comunicación, negocios y compromiso organizacional, todos relacionados al subtema de ciencias de administración y gestión (Ver tabla 2).

Tabla 1: Número de artículos por autores de la revista Innova Research Journal en el 2016

\begin{tabular}{cccccccc}
\hline Mes & Publicaciones & & & & Autores & & \\
& & Simple & Doble & Triple & Cuádruple & Quíntuple & Séxtuple \\
\hline 1 Trimestre & 15 & 11 & 3 & 0 & 0 & 1 & 0 \\
2 Trimestre & 15 & 13 & 1 & 0 & 0 & 0 & 1 \\
3 Trimestre & 19 & 9 & 4 & 2 & 1 & 2 & 1 \\
4 Trimestre & 41 & 13 & 15 & 9 & 3 & 0 & 1 \\
Total & 90 & 46 & 23 & 11 & 4 & 3 & 3 \\
\hline
\end{tabular}

Fuente: Revista Innova Research Journal, 2016.

Tabla 2: Keywords más utilizadas en publicaciones de la revista Innova Research Journal

\begin{tabular}{lr}
\hline Palabras claves & 13 \\
\hline Liderazgo & 5 \\
Comunicación & 5 \\
Negocios & 4 \\
Compromiso organizacional &
\end{tabular}

Fuente: Revista Innova Research Journal, 2016.

La producción de contenido por número de páginas de 4 a 55 páginas con una diferencia de longitud de publicaciones, donde 10 artículos escribieron 10 páginas (11\%) (Ver tabla 3).

Tabla 3: Contenido de páginas producidas de los artículos en el 2016 de la revista Innova Research Journal

\begin{tabular}{cccc}
\hline Número de páginas & Número de artículos & Porcentaje & Páginas producidas \\
\hline $\mathbf{1 2}$ & 10 & $11 \%$ & 120 \\
$\mathbf{6}$ & 9 & $10 \%$ & 54 \\
$\mathbf{1 3}$ & 9 & $10 \%$ & 117 \\
$\mathbf{8}$ & 8 & $9 \%$ & 64 \\
$\mathbf{7}$ & 6 & $7 \%$ & 42 \\
$\mathbf{9}$ & 6 & $7 \%$ & 54 \\
$\mathbf{4}$ & 5 & $6 \%$ & 20 \\
$\mathbf{5}$ & 5 & $6 \%$ & 25 \\
$\mathbf{1 1}$ & 5 & $6 \%$ & 55 \\
$\mathbf{1 8}$ & 4 & $4 \%$ & 72 \\
$\mathbf{1 0}$ & 4 & $4 \%$ & 40 \\
$\mathbf{1 9}$ & 3 & $3 \%$ & 57 \\
$\mathbf{1 4}$ & 3 & $3 \%$ & 42 \\
$\mathbf{1 5}$ & 3 & $3 \%$ & 45 \\
\hline
\end{tabular}




\begin{tabular}{cccc}
\hline $\mathbf{2 1}$ & 2 & $2 \%$ & 42 \\
$\mathbf{1 6}$ & 2 & $2 \%$ & 32 \\
$\mathbf{1 7}$ & 2 & $2 \%$ & 34 \\
$\mathbf{2 0}$ & 2 & $2 \%$ & 40 \\
$\mathbf{5 5}$ & 1 & $1 \%$ & 55 \\
$\mathbf{2 3}$ & 1 & $1 \%$ & 23 \\
& 90 & & 1033 \\
\hline
\end{tabular}

Fuente: Revista Innova Research Journal, 2016.

La tabla 4 indica los artículos publicados en relación a la palabra clave liderazgo, la más mencionada, se encuentra la participación de Guerrero Bejarano y Nájera Santiago

Tabla 4: Artículos publicados con el keyword más declarados (liderazgo) en la revista Innova Research Journal

\begin{tabular}{|c|c|c|}
\hline Artículos & Autor & Coautor \\
\hline $\begin{array}{l}\text { Desarrollo de soft skills una } \\
\text { alternativa a la escasez de talento } \\
\text { humano }\end{array}$ & Serrano Orellana Bill & Tito Maya María \\
\hline $\begin{array}{l}\text { El Contrato Psicológico y su relación } \\
\text { con el Compromiso Organizacional }\end{array}$ & Guerrero Bejarano María & \\
\hline $\begin{array}{l}\text { El gran salto: De vendedor a } \\
\text { supervisor }\end{array}$ & Paz y Miño Mario & \\
\hline $\begin{array}{l}\text { El Pensamiento Crítico como una } \\
\text { herramienta de desarrollo de } \\
\text { Liderazgo }\end{array}$ & Guerrero Bejarano María & Medina Vergara Viviana \\
\hline $\begin{array}{l}\text { - El Rol de la Ética en los Estilos de } \\
\text { Liderazgo }\end{array}$ & Nájera Santiago & \\
\hline - Integrando el pensamiento critico & Nájera Santiago & \\
\hline - Inteligencia Emocional y Liderazgo & Brito Jorge G. & \\
\hline $\begin{array}{l}\text { La Gestión del Conocimiento en las } \\
\text { Empresas, su importancia y } \\
\text { dependencia del Estilo del Liderazgo } \\
\text { de la Alta Gerencia }\end{array}$ & Guerrero Bejarano María & \\
\hline $\begin{array}{l}\text { La influencia de la personalidad en } \\
\text { los estilos de liderazgo }\end{array}$ & Méndez Bravo Julio Cesar & \\
\hline $\begin{array}{l}\text { La Relación del Capital Psicológico } \\
\text { Cultural y el Global Mindset en el } \\
\text { Contexto XYZ. }\end{array}$ & Fierro Isidro & \\
\hline $\begin{array}{l}\text { La Relación entre los Estilos de } \\
\text { Liderazgo, la Satisfacción Laboral y } \\
\text { su Efecto en el Compromiso } \\
\text { Organizacional }\end{array}$ & Guerrero Bejarano María & \\
\hline - Liderazgo e inteligencia emocional & Nájera Santiago & \\
\hline $\begin{array}{l}\text { Liderazgo, Creatividad y } \\
\text { Pensamiento Crítico }\end{array}$ & Nájera Santiago & \\
\hline
\end{tabular}

Fuente: Revista Innova Research Journal, 2016.

La tabla 5 muestra los autores con mayor participación fueron Guerrero Bejarano María, Nájera Santiago, Chávez Eras Andrés, Brito Jorge, seguido por Maino Isaías Aldo, Jurado Eduardo, Noroña Marco con 3 publicaciones cada uno. Con 2 artículos 5 autores, además 12 
autores con participación como autores y coautores, seguidos 6 autores con participación de coautores. Con 1 artículo, finalmente 42 autores con una participación como autor y 53 auoteres solo con participación como coautores.

Tabla 5: Autores con participación en el 2016 en la revista Innova Research Journal

\begin{tabular}{lrrr}
\hline \multicolumn{1}{c}{ Participantes } & Autor & Coautor & Total general \\
\hline Guerrero Bejarano María & 6 & & 6 \\
Nájera Santiago & 5 & & 5 \\
Chávez Eras Andrés & 4 & 1 & 5 \\
Brito Jorge G. & 4 & & 4 \\
Maino Isaías Aldo & 3 & & 3 \\
Jurado V Eduardo & 2 & 1 & 3 \\
Noroña Merchán Marco Vinicio & 1 & 2 & 3 \\
Pico Lucía Magdalena & 2 & & 2 \\
Jordán Correa Dennys & 2 & & 2 \\
Ramos Rivero Virgilio Lucas & 2 & & 2 \\
Gavilondo Rodríguez Carlos Ernesto & 2 & & 2 \\
Abrigo Córdova Pablo Antonio & 2 & & 2 \\
Rojas Bajaña Roberto Andrés & 1 & 1 & 2 \\
Encalada Noboa Jorge & 1 & 1 & 2 \\
Silva Peñaherrera & 1 & 1 & 2 \\
Gómez García Ramón & 1 & 1 & 2 \\
Armijos Acosta Rosa Miriam & 1 & 1 & 2 \\
Herrera Rivas Keila Ketty & 1 & 1 & 2 \\
Vergara Díaz Nelly & 1 & 1 & 2 \\
Pavón Brito Christian & 1 & 1 & 2 \\
Brito Ochoa María Paulina & 1 & 1 & 2 \\
Yangali Vicente Judith Soledad & 1 & 1 & 2 \\
Saltos Aldaz Luis Alberto & 1 & 1 & 2 \\
Barrios Queipo Ronald Álvarez & 1 & 1 & 2 \\
San Andrés Reyes Pablo & & 2 & 2 \\
Vilaret Serpa & & 2 & 2 \\
Serrano Orellana Bill & & 2 & 2 \\
Morales Neira David Job & & & 2 \\
Suasnavas Bermúdez & & & 2 \\
Murillo Salazar Marcos Genaro & & & 2 \\
\hline & & & 2 \\
\hline
\end{tabular}

Fuente: Revista Innova Research Journal, 2016.

En el desarrollo de las publicaciones son importantes las referencias o fuentes bibliográficas que sustenta el enfoque introductorio, metodológico o las conclusiones. Para este análisis bibliotecológico de la revista INNOVA del 2016 se presenta 1970 fuentes declaradas en las publicaciones de las cuales en dos artículos presenta 2 bibliografías y un artículo con 106 fuentes contando con 9 artículos en inglés, como se observa en la Tabla 6 se agrupó en rangos de la cantidad de fuentes bibliográficas de las publicaciones. 
Tabla 6: Rango de cantidad de fuentes bibliográficas de las publicaciones en la revista Innova Research Journal

\begin{tabular}{lcc}
\hline \multicolumn{1}{c}{ Rango } & Publicaciones & $\%$ \\
\hline $\mathbf{2 - 1 2}$ & 34 & $38 \%$ \\
$\mathbf{1 3 - 2 3}$ & 26 & $29 \%$ \\
$\mathbf{2 4 - 3 4}$ & 17 & $19 \%$ \\
$\mathbf{3 5 - 4 5}$ & 4 & $4 \%$ \\
$\mathbf{4 6 - 5 6}$ & 2 & $2 \%$ \\
$\mathbf{5 7 - 6 7}$ & 3 & $3 \%$ \\
Mayor a 68 & 4 & $4 \%$ \\
& 90 & $100 \%$
\end{tabular}

Fuente: Revista Innova Research Journal, 2016.

Basado en los datos fluctuantes de análisis de tendencia polinómica de orden 2 en relación de la cantidad de fuentes bibliográficos de las publicaciones se observa que el valor $\mathrm{R}$ cuadrado es 0,9687 comprende un ajuste de los datos.

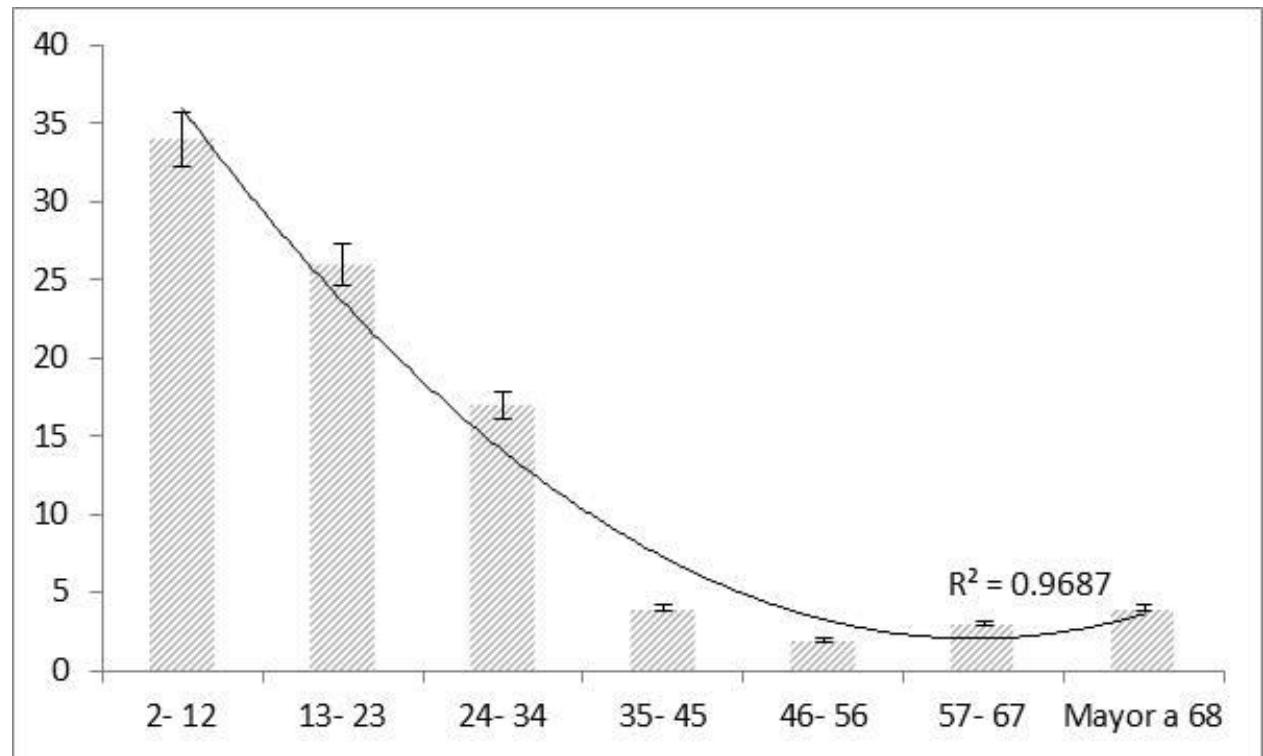

Figura 1: Tendencia polinómica de las fuentes de las publicaciones en la revista Innova Fuente: Revista Innova Research Journal, 2016.

Se analizó como parámetro cuanto ha sido referenciado los artículos por otros autores para esto se utilizó google scholar de todos los artículos de INNOVA, como se presenta en la Tabla 7 corresponde 7 artículos citados. 
Tabla 7: Análisis de referencias bibliográficas por autores y citas revista Innova Research Journal

\begin{tabular}{|c|c|c|c|}
\hline Artículos & Keyword más mencionada & Autor mayor participación & Citado \\
\hline 1. Guerrero, B. M. A.(2016). La & $\mathrm{X}$ & $\mathrm{X}$ & 1 \\
\hline \multicolumn{4}{|l|}{ Investigación Cualitativa. INNOVA } \\
\hline \multicolumn{4}{|l|}{ Research Journal, 1(2), 1-9. } \\
\hline 2. Nájera, S. (2016). Teoría de Juegos & $\mathrm{X}$ & $\mathrm{X}$ & 1 \\
\hline \multicolumn{4}{|l|}{ y Gestión Estratégica. INNOVA } \\
\hline \multicolumn{4}{|l|}{ Research Journal, 1(1), 25-33. } \\
\hline $\begin{array}{l}\text { 3. Brito, J. G. (2016). La aplicaciones } \\
\text { de la teoría de juegos en la gerencia } \\
\text { estratégica. INNOVA Research Journal, } \\
\text { 1(5), 9-13. }\end{array}$ & & $\mathrm{X}$ & 1 \\
\hline \multicolumn{4}{|l|}{$\begin{array}{l}\text { 4. Abrigo, P. (2016). La fertilización } \\
\text { orgánica del melloco (Ullucus tuberosus }\end{array}$} \\
\hline \multicolumn{4}{|l|}{ L.) y su rentabilidad en la comunidad de } \\
\hline \multicolumn{4}{|l|}{ Tuncarta. INNOVA Research Journal, 1 , } \\
\hline 5. Abrigo C. P. A. (2016). & & & 2 \\
\hline \multicolumn{4}{|l|}{ Identificación de Necesidades para } \\
\hline \multicolumn{4}{|l|}{ Proponer el Desarrollo Turístico en la } \\
\hline \multicolumn{4}{|l|}{ Comunidad de Tuncarta, del Cantón } \\
\hline \multicolumn{4}{|l|}{ Saraguro de la Provincia de Loja. } \\
\hline \multicolumn{4}{|l|}{ INNOVA Research Journal, 1(6), 27-41. } \\
\hline 6. Garcés, N. N. G. (2016). La & & & 1 \\
\hline \multicolumn{4}{|l|}{ inversión acertada en la Educación } \\
\hline \multicolumn{4}{|l|}{ Superior como desarrollo sostenible. } \\
\hline \multicolumn{4}{|l|}{ INNOVA Research Journal, 1(8), 50-56. } \\
\hline 7. Benavente, M. A. (2016). Análisis & & & 1 \\
\hline de la Inversión Directa China en & & & \\
\hline Ecuador en el Período 2000 a 2014. & & & \\
\hline $\begin{array}{l}\text { INNOVA Research Journal, 1(10), 67- } \\
79 .\end{array}$ & & & \\
\hline
\end{tabular}

Fuente: Revista Innova Research Journal, 2016.

\section{Conclusión}

Uno de los objetivos del Reglamento de Régimen Académico del Ecuador es "articular la formación académica científica, tecnológica y social y la vinculación con la colectividad, en un marco de calidad, innovación y pertinencia" (CES, 2013) y ante los retos de la era digital la función del docente no solo es de transmitir sino de ayudar para aprender en la construcción del conocimiento. (Pérez, 2012).

El trabajo de investigación se centra en una opinión bibliográficamente de las publicaciones en la revista INNOVA e identificar una prospectiva y tendencia del objeto de estudio, los resultados presentan un crecimiento importante de artículos principalmente en temas de administración y educación; para los resultados de la investigación se sugiere el abordaje de aportar al manejo de la información, no solo de crear artículos también el impulsar aspectos académicos dentro de las aulas universitarias adoptando algún modelo de aprendizaje que involucre opinar y evaluar la investigación elaborada, pues es constante cumplir con las actividades académicas, para lograr estudiantes motivados y desarrollar el interés por cumplir 
con las actividades asignadas para generar momentos críticos a los procesos de investigación (Correa. Et al, 2017).

Para este trabajo se contó con todos los artículos del 2016 de la revista elaborándose una base de datos con el nombre de autores, palabras claves, número de páginas, referencias bibliográficas citadas, idioma, citaciones en google scholar, obteniéndose una alta participación de los autores Guerrero Bejarano Marís y Nájero Santiago.

\section{Bibliografía}

Abrigo, P. (2016). La fertilización orgánica del melloco (Ullucus tuberosus L.) y su rentabilidad en la comunidad de Tuncarta. INNOVA Research Journal, 1, 12.

Acosta, R. A., \& Rivas, K. K. H. (2016). Apreciación artística: Herramienta interdisciplinaria para un desarrollo cognitivo en la educación. INNOVA Research Journal, 1(12), 29-34.

Acosta, R. M. A., \& Rivas, K. K. H. (2016). Arte y lectura vinculos entre universidad y comunidad. INNOVA Research Journal, 1(11), 141-145.

Aguilar, G. F. C., Pupo, I. P., Pérez, P. P., Sosa, R., Vicet, P. V., \& Bermúdez, M. V. (2016). PRODANALYSIS, un Sistema para el Aseguramiento de Ingresos Basado en Minería de Outliers. INNOVA Research Journal, 1(7), 18-36.

Aguilera, C. L. R. (2016). Breve análisis del intercambio comercial de Venezuela y el MERCOSUR: 2006-2015. INNOVA Research Journal, 1(9), 1-12.

Aguirre, C. A. S., Sares, B. C. M., \& Orellana, B. J. S. (2016). El Emprendimiento Como Base En La Condición Socioeconómica. INNOVA Research Journal, 1(12), 1-10.

Aldaz, A. S., Neira, D. M., Bajaña, R. R., \& Salazar, M. M. (2016). Breve análisis del Proyecto Educativo "Deportes del Buen Vivir" aplicado en la Unidad Educativa Liceo Naval de Guayaquil periodos lectivos 2014-2015, 2015-2016. INNOVA Research Journal, 1(6), 49-61.

Anchundia, M. E. O., \& Ulloa, P. L. (2016). Análisis de la Interacción en Twitter, entre los televidentes y los noticieros matinales en la ciudad de Guayaquil (2015). INNOVA Research Journal, 1(9), 58-76.

Arévalo, B., \& Ninibeth, E. (2016). Análisis de la difusión de programas educativos en los medios de comunicación del cantón Vinces, período julio 2014-julio 2015.

Asamblea Constituyente. (2008). Constitución del Ecuador. Obtenido de http://www.asambleanacional.gov.ec/documentos/constitucion_de_bolsillo.pdf

Bajaña, R. A. R., Neira, D. J. M., Aldaz, L. A. S., Salazar, M. M., \& Mora, A. B. C. (2016). Valoración de la unidad didáctica de sensibilización "Todos al agua" para diversidad funcional asociada con discapacidad cognitiva "Síndrome de Down”. INNOVA Research Journal, 1(8), 36-49. 
Balás, J. E. (2016). Retiro Estudiantil Salesiano:¿ quién es culpable: la universidad, la metodología docente o el desempeño estudiantil?. INNOVA Research Journal, 1(9), 114-136.

Baquerizo, J. I. C., Baquerizo, D. R. C., \& Cristóbal, L. R. A. (2016). Factores Determinantes de la Eficiencia de la Gestión Administrativa en las Instituciones de Educación Superior. INNOVA Research Journal, 1(11), 146-156.

Bejarano, M. A. G. (2016). El Contrato Psicológico y su relación con el Compromiso Organizacional. INNOVA Research Journal, 1(12), 44-51.

Bejarano, M. A. G. (2016). La Investigación Cualitativa. INNOVA Research Journal, 1(2), 1-9.

Bejarano, M. A. G. (2016). La Relación entre los Estilos de Liderazgo, la Satisfacción Laboral y su Efecto en el Compromiso Organizacional. INNOVA Research Journal, 1(10), 134-144.

Bejarano, M. A. G., \& Cobeña, J. A. V. (2016). La importancia de la asociatividad para el desarrollo. INNOVA Research Journal, 1(11), 105-119.

Bejarano, M. A. G., \& Siu, D. R. S. (2016). La Gestión del Conocimiento y los Sistemas de Información como fuentes de Ventaja Competitiva para las Empresas. INNOVA Research Journal, 2(4), 73-76.

Bejarano, M. A. G., \& Siu, D. R. S. (2017). La Gestión del Conocimiento y los Sistemas de Información como fuentes de Ventaja Competitiva para las Empresas. INNOVA Research Journal, 2(4), 73-76

Bejarano, M. A. G., \& Vergara, V. V. M. (2016). El Pensamiento Crítico como una herramienta de desarrollo de Liderazgo. INNOVA Research Journal, 1(3), 1-4.

Benavente, M. A. (2016). Análisis de la Inversión Directa China en Ecuador en el Período 2000 a 2014. INNOVA Research Journal, 1(10), 67-79.

Bravo, J. C. M. (2016). La influencia de la personalidad en los estilos de liderazgo. INNOVA Research Journal, 1(12), 52-58.

Brito, C. P., Noboa, J. E., \& Fernandez, K. M. (2016). Página web para fortalecer el conocimiento general de la Física: un ejemplo de proyecto semillero. INNOVA Research Journal, 1(12), 2328.

Brito, J. G. (2016). Inteligencia emocional y liderazgo. INNOVA Research Journal, 1(4), 16-21.

Brito, J. G. (2016). La aplicaciones de la teoría de juegos en la gerencia estratégica. INNOVA Research Journal, 1(5), 9-13.

Brito, J. G. (2016). La Ética y Los Estilos de Liderazgo. INNOVA Research Journal, 1(2), 41-48.

Brito, J. G. (2016). Personalidad y liderazgo. INNOVA Research Journal, 1(3), 76-83. 
Calvache, M. B. (2016). La regionalización sudamericana en le marco de la adopción e impementación de la Televisión Digital Terrestre. INNOVA Research Journal, 1(10), 21-36.

Camacho, E. L. (2016). Elementos pedagógicos significativos del discurso de Jesucristo. INNOVA Research Journal, 1(7), 6-17.

Carrillo, F. A. H., Flores, N. C. C., \& Esteves, T. B. C. Q. (2016). Modelos de Satisfacción: Fundamentación Teórica y Criterios de Aplicación. INNOVA Research Journal, 1(10), 145-155.

Castro, S. M. (2016). La posibilidad de extender los derechos humanos a la empresa: ¿ Una opción posible?. INNOVA Research Journal, 1(9), 39-57.

CEAACES. (2015). http://www.ceaaces.gob.ec. Obtenido de http://www.ceaaces.gob.ec: http://www.ceaaces.gob.ec/sitio/wp-content/uploads/2013/10/Adaptacio\%Cc\%81n-DelModelo-De-Evaluacio\%Cc\%81n-Institucional-De-Univ.-Y-Esc.-Polite\%Cc\%81c.-2013-AlProceso-De-Eval-Acredit-Y-Recateg-De-Univers.-Y-Esc.-Polit-2015plenofinal-Notif.pdf

Chauí citado por Guimar' es Pompìo de Camargo, María Valencia. 3 Pesquisador científico: Avaliação de produçao 1990/1994 3 En: Porto Witter, G. Produção científica. Campinas, SP: Editora Á tomo, 1997. p. 237

Chong, M. E. B. (2016). Análisis del modelo de desarrollo organizacional de las empresas públicas anexas al gobierno autónomo descentralizado de Manabí, para promover proyectos de desarrollo socio económico en la provincia de Manabí. INNOVA Research Journal, 1(10), 92-103.

Córdova, P. A. A. (2016). Identificación de Necesidades para Proponer el Desarrollo Turístico en la Comunidad de Tuncarta, del Cantón Saraguro de la Provincia de Loja. INNOVA Research Journal, 1(6), 27-41.

Correa, D. J. (2016). Club de Periodismo, modelo de práctica educativa para fortalecer la escritura periodística. INNOVA Research Journal, 1(6), 14-26.

Correa, D. J., \& Dominguez, J. A. (2016). La crónica, el género literario del periodismo que merece sobrevivir. INNOVA Research Journal, 1(12), 35-43.

Correa, M., Vinueza, J., Torres A., E. \& Ponce I., K. (2017). Sistema para las tutorías académicas en las universidades ecuatorianas. Caso Universidad Estatal de Milagro. INNOVA Research Journal, 2(6), 100-11.

Diaz, N. S. V., Intriago, F. R. O., Tomalá, V. H. V., Lopez, D. A. M., Paredes, D. A. B., \& Reyes, P. R. S. A. (2016). El cultivo de soya y su importancia para el Ecuador. INNOVA Research Journal, l(12), 77-85.

Eras, A. C. (2016). Bolsa familia: inequality and education in brazil. INNOVA Research Journal, 1(3), 5-12.

Eras, A. C. (2016). Development Ideas Changing the World. INNOVA Research Journal, 1(4), 5-9. 
Eras, A. C. (2016). Primary Education in Nigeria at a Glance: Statistical Overview of the Subsector. INNOVA Research Journal, 1(9), 96-113.

Eras, A. C., Joseph, N., Franco, L., Leng, P., \& Pierre, G. (2016). The World Bank's Education For All Phase II in Haiti Case Study. INNOVA Research Journal, 1(2), 10-27.

Fierro, I. (2016). La Relacion del Capital Psicologico Cultural y el Global Mindset en el Contexto XYZ. INNOVA Research Journal, 1(7), 1-5.

Gallego Becerra, Hugo Armando; Rodríguez-Morales, Alfonso J.; (2013). La publicación científica y sus paradigmas. Scientia Et Technica, Octubre-

Garcés, N. N. G. (2016). La inversión acertada en la Educación Superior como desarrollo sostenible. INNOVA Research Journal, 1(8), 50-56.

García, A. R. G. (2016). Propuesta metodológica para el establecimiento de líneas de investigación en seguridad y salud en el trabajo. INNOVA Research Journal, 1(9), 13-25.

Guerra, Y. P. (2016). Fortalecimiento de la gestión empresarial en las pequeñas y medianas empresas en Ecuador, a partir del uso de herramientas cualitativas y cuantitativas. INNOVA Research Journal, $1(10), 49-66$.

Isaias, A. M. (2016). Environmental Adjustment, Academic Involvement and Difficulties of Students with Disabilities in Ecuador. INNOVA Research Journal, 1(2), 34-40.

Isaias, A. M. (2016). Marketing Strategies in Enrollment Management: Direct Mail and Financial Aid. INNOVA Research Journal, 1(1), 34-39.

Isaias, A. M. (2016). The Impact of Student Communities on Student Development: Institutional Size and Residential Living. INNOVA Research Journal, 1(4), 22-30.

Jurado, E. (2016). Administrando el Cambio. INNOVA Research Journal, 1(2), 28-33.

Jurado, E., \& Cedeño, A. R. (2016). El flujo de caja libre, operativo y del accionista, los elementos creadores de valor, los value drivers o inductores de valor corporativos, relaciones entre el FCF. INNOVA Research Journal, 1(3), 21-75.

Jurado, T. C., \& Rosero, R. S. (2016). Evolución del Modelo Premo para determinar las emociones de marca basada en las percepciones. INNOVA Research Journal, 1(7), 47-58.

Latindex. (2015). http://www.latindex.org. Obtenido de http://www.latindex.org: http://www.latindex.org/latindex/Solar/Busqueda

Lukomski, A. (2016). La verdad como valor integrador desde el pensamiento complejo de Santo Tomas de Aquino. INNOVA Research Journal, 1(6), 42-48.

Macias, M. F. M. (2017). Las TICS y su utilización para la lectura, la linguistica y la literatura como tools generadoras de competencias en los discentes del milenio. INNOVA Research Journal, 1(12), $150-156$. 
Martínez, E. J. F. (2016). La gestión del departamento de talento humano en la capacitación y desarrollo del profesorado ecuatoriano. INNOVA Research Journal, 1(11), 120-128.

Maya, M. T., \& Orellana, B. S. (2016). Desarrollo de soft skills una alternativa a la escasez de talento humano. INNOVA Research Journal, 1(12), 59-76.

Mediavilla, J. J. C. (2016). Diseño y construcción de colectores de admisión de aire con tomas individuales para cada cilindro de un motor. INNOVA Research Journal, 1(12), 118-131.

Ministerio de Educación del Ecuador. (2012). Suplemento del Registro Oficial No. 955, de 3 de Marzo de 2017. Obtenido de http://educaciondecalidad.ec/ley-educacion-interculturalmenu/reglamento-loei-texto.html

Morales-Morejon; M, Morales-Aguilera. M. La informetría y las fuentes de información personales e institucionales. Su importancia en relación con la información de inteligencia. Ciencias de la información. (La Habana) 28(3):207-217, septiembre 1997.

Nájera, S. (2016). El Rol de la Ética en los Estilos de Liderazgo. INNOVA Research Journal, 1(5), 1418.

Nájera, S. (2016). Integrando el pensamiento critico. INNOVA Research Journal, 1(3), 13-20.

Nájera, S. (2016). Liderazgo e Inteligencia Emocional. INNOVA Research Journal, 1(1), 19-24.

Nájera, S. (2016). Liderazgo, creatividad y pensamiento crítico. INNOVA Research Journal, 1(4), 1-4.

Nájera, S. (2016). Teoría de Juegos y Gestión Estratégica. INNOVA Research Journal, 1(1), 25-33.

Neira, H. (2016). Tengo una idea de negocio.¿ Ahora qué?. INNOVA Research Journal, 1(5), 19-22.

Noboa, J. E., \& Brito, C. P. (2017). Laboratorios Virtuales: una alternativa para mejorar el rendimiento de los estudiantes y la optimización de recursos económicos. INNOVA Research Journal, 1(11), 9196.

Noroña, M. V. (2016). Diseño y factibilidad de una planta de ensambles de tableros de instrumentos del vehículo aplicado en el Ecuador. INNOVA Research Journal, 1(9), 137-157.

Ochoa, M. P. B. (2016). Análisis comparativo del impacto de la transversalidad de la cátedra de emprendimiento en la disposición para nuevos emprendimientos de los alumnos de la Universidad de Guayaquil y la Escuela Superior Politécnica del Litoral. INNOVA Research Journal, 1(10), 3748.

Orozco, C. A. C. (2016). Síndrome de Burnout en docentes universitarios. INNOVA Research Journal, 1(9), 77-95.

Paz, M. (2016). El gran salto: De vendedor a supervisor. INNOVA Research Journal, 1(5), 5-8. 
Peñafiel, M. A. V. (2016). Impacto del semáforo nutricional en los hábitos alimenticios de los estudiantes de la Universidad Politécnica Salesiana. INNOVA Research Journal, 1(10), 80-91.

Pérez Rojas, R. L. (2012). Universidad Veracruzana. Obtenido de http://www.uv.mx/dgdaie/files/2012/11/z-El-sistema-tutorial-en-la-UV.pdf

Pico, L. (2016). Calidad y Desarrollo en la Educación a Distancia. INNOVA Research Journal, 1(5), $1-4$.

Pico, L. M. (2016). La gestión del talento humano, recurso indispensable para la organización en el entorno competitivo actual. INNOVA Research Journal, 1(11), 97-104.

Piedra Salomón, Yelina; Martínez Rodríguez, Ailín; (2007). Producción científica. Ciencias de la Información, Diciembre-Sin mes, 33-38.

Queipo, E. A. B., Buenafé, A. A., \& Jiménez, N. M. R. (2016). Concepción metodológica del diseño y rediseño de carreras en la Universidad Internacional SEK Ecuador. INNOVA Research Journal, $1(10), 104-114$.

Reyes, P. R. S. A., Farfán, E. E. C., Terán, P. J. P., \& Díaz, N. S. V. (2016). Factores claves para la viabilidad de emprendimientos gastronómicos en momentos de crisis, en Guayaquil. INNOVA Research Journal, 1(11), 63-70.

Rivera, P. L. G. (2016). Capacitación Profesoral y Didáctica Universitaria. INNOVA Research Journal, $1(11), 30-41$.

Rivero, V. L. R., \& Pinargote, A. J. P. (2016). Consideraciones para una metodología de trabajo pericial en el campo automotriz. INNOVA Research Journal, 1(12), 86-102.

Rivero, V. L. R., \& Rendón, R. A. P. (2016). Indicadores para valorar el grado de significación de los aprendizajes en las asignaturas de formación profesional. INNOVA Research Journal, 1(8), 28-35.

Rodas, B. I., \& Álvarez, R. (2016). Influencia de la página de Facebook de la Universidad Politécnica Salesiana, sede Guayaquil, como medio de información para los estudiantes. INNOVA Research Journal, 1(11), 42-62.

Rodríguez, C. E. G. (2016). El soporte video como apoyo al proceso de enseñanza. INNOVA Research Journal, 1(6), 1-13.

Rodríguez, C. E. G., \& Ospina, J. M. P. (2016). La creación de productos comunicativos audiovisuales. Una mirada al audiovisual ecuatoriano. INNOVA Research Journal, 1(9), 26-38.

Rodríguez, M. M. F. (2016). Fundamentos Filosóficos y Sociológicos de la Inclusión Educativa y la Atención a la Diversidad. INNOVA Research Journal, 1(10), 115-120.

Rodriguez, V. E. (2016). Estrategia metodológica para desarrollar la competencia del manejo de la información en estudiantes universitarios. INNOVA Research Journal, 1(11), 157-168. 
Rovayo, M. E. D., \& Eras, A. C. (2016). Status of Lifelong Learning in Guayaquil, Ecuador. INNOVA Research Journal, 1(10), 121-133.

Salazar, G. M. D., Alvarado, E. S. Q., Franco, P. I. V., \& Ochoa, M. P. B. (2016). El dinero electrónico como medio de pago para la inclusión tributaria en Ecuador. INNOVA Research Journal, 1(10), 111.

Sancan, D. G. C., \& Merchán, M. V. N. (2016). Modelado e implementación del tractor john deere 6125d en la preparación de la tierra para cultivos de arroz. INNOVA Research Journal, 1(12), 103117.

Skeef citado por Mollo Pécora, Gláncia.3 Actividades académicas de pesquisador En: Porto Witter, G. Produção científica. Campinas, SP: Editora Á tomo, 1997. p.158

Sotomayor, V. A. M., Betancourt, D., \& Sangurima, W. J. (2016). Diseño de Ciclovías para Ciudades Intermedias, una propuesta para la Ciudad de Loja. INNOVA Research Journal, 1(12), 11-22.

Torres, M. G., \& Merchán, M. V. N. (2016). Diseño e implementación de máquina cargadora frontal sobre ruedas 1120 e volvo para la limpieza y remoción de tierra por deslaves en la vía aloag-santo domingo. INNOVA Research Journal, 1(11), 71-90.

Vasquez, M. E. (2016). La Banca Privada en el Crecimiento Económico de un país. INNOVA Research Journal, 1(4), 10-15.

Vicente, J. S. Y. (2016). La gestion educativa en el desarrollo de la calidad universitaria. INNOVA Research Journal, 1(7), 37-46.

Vicente, J. S. Y., \& López, J. L. R. (2016). Aplicación del Método PÓLYA para Mejorar el Rendimiento Académico de Matemática en los Estudiantes de Secundaria. INNOVA Research Journal, 1(10), 12-20.

Villamagua, J. S. B. (2016). Loja como icono en las artes vivas, sobre sus potencialidades y posibilidades para el festival internacional. INNOVA Research Journal, 1(11), 1-16.

Vinueza, E. J., \& Jaramillo, E. (2016). Marketing Internacional. INNOVA Research Journal, 1(1), 818.

Wahnoun, C. R. (2016). Édouard Glissant's work, an entrance towards the "Relation": intentions and projections. INNOVA Research Journal, 1(11), 129-140.

Yánez, P. (2016). Factores socio-ambientales y de conservación en predios amazónicos de Ecuador vinculados o no al Programa Socio Bosque. INNOVA Research Journal, 1(11), 17-29.

Zotta, Claudio Marcelo; (2015). Porque es importante la publicación científica. Journal of the Selva Andina Research Society . 